\title{
Characterization of probiotic Escherichia coli isolates with a novel pan-genome microarray Hanni Willenbrock ${ }^{* \dagger}$, Peter F Hallin*, Trudy M Wassenaar ${ }^{* \ddagger}$ and David W Ussery*
}

\begin{abstract}
Addresses: *Center for Biological Sequence Analysis, Technical University of Denmark, 280o, Lyngby, Denmark. ${ }^{\dagger}$ Exiqon A/S, 2950 Vedbæk,
\end{abstract} Denmark. ${ }^{*}$ Molecular Microbiology and Genomics Consultants, Tannenstrasse, 55576 Zotzenheim, Germany.

Correspondence: Hanni Willenbrock. Email: hanni@cbs.dtu.dk

Published: 18 December 2007

Genome Biology 2007, 8:R267 (doi:10.1 186/gb-2007-8-12-r267)

The electronic version of this article is the complete one and can be found online at http://genomebiology.com/2007/8/I2/R267
Received: 30 July 2007

Revised: 4 October 2007

Accepted: 18 December 2007

(C) 2008 Willenbrock et al.; licensee BioMed Central Ltd.

This is an open access article distributed under the terms of the Creative Commons Attribution License (http://creativecommons.org/licenses/by/2.0), which permits unrestricted use, distribution, and reproduction in any medium, provided the original work is properly cited.

\begin{abstract}
Background: Microarrays have recently emerged as a novel procedure to evaluate the genetic content of bacterial species. So far, microarrays have mostly covered single or few strains from the same species. However, with cheaper high-throughput sequencing techniques emerging, multiple strains of the same species are rapidly becoming available, allowing for the definition and characterization of a whole species as a population of genomes - the 'pan-genome'.

Results: Using 32 Escherichia coli and Shigella genome sequences we estimate the pan- and core genome of the species. We designed a high-density microarray in order to provide a tool for characterization of the $E$. coli pan-genome. Technical performance of this pan-genome microarray based on control strain samples (E. coli K-12 and O157:H7) demonstrated a high sensitivity and relatively low false positive rate. A single-channel analysis approach is robust while allowing the possibility for deriving presence/absence predictions for any gene included on our pan-genome microarray. Moreover, the array was highly sufficient to investigate the gene content of nonpathogenic isolates, despite the strong bias towards pathogenic $E$. coli strains that have been sequenced so far.
\end{abstract}

Conclusion: This high-density microarray provides an excellent tool for characterizing the genetic makeup of unknown $E$. coli strains and can also deliver insights into phylogenetic relationships. Its design poses a considerably larger challenge and involves different considerations than the design of single strain microarrays. Here, lessons learned and future directions will be discussed in order to optimize design of microarrays targeting entire pan-genomes. 


\section{Background}

Bacterial isolates are traditionally classified into species by bacteriological methods, and subtyped within the species by phenotypic or genotypic characterization. For the identification and subtyping of Escherichia coli isolates, a wide variety of typing methods have been developed. A recent addition to this spectrum is array comparative genomic hybridization (aCGH) [1]. Thus, microarray hybridization is becoming a standard procedure to evaluate the genetic content of a bacterial species. For E. coli, a microarray covering the gene content of seven strains was recently developed for the characterization of emerging pathogens [2]. However, since then, many additional E. coli strains and plasmids have been sequenced, and the total number of genes potentially present in E. coli strains, the so-called 'pan-genome' [3,4], increases with each new $E$. coli genome sequenced. A microarray chip approximating the complete pan-genome of $E$. coli would provide optimal sensitivity to characterize isolates. Here, we present a novel design of a microarray covering the complete currently known genome content of 32 sequenced genomes. Such a pan-genome microarray can be used for more precise characterization of novel strains, including emerging pathogens, and can also deliver insights into phylogenetic relationships.

Phylogenetic relationships are commonly determined by bacterial subtyping. Due to the complex sexual behavior of bacteria, phylogenetic trees obtained with individual genes often do not correspond to each other. Although multilocus sequence typing is now regarded by many as a good standard to determine phylogenetic relationships between and within bacterial species, it does not always reflect the true genetic diversity of members of a species; trees based on multilocus sequence typing may, therefore, differ significantly from a tree based on whole gene content [3]. A pan-genome microarray may offer a suitable alternative to complete genome sequencing for extracting the necessary gene content to construct a realistic phylogenetic tree based on conserved gene content. The recent technological development in sequencing and the consequent price drop have led to an explosion of available genome sequences and perhaps within a few years will lead to sequencing being a faster and cost effective alternative to CGH microarray analysis. However, at the moment, sequencing is still more costly and less time efficient than hybridization experiments, while hybridization experiments potentially also can provide information regarding gene expression.

Here, we determine an approximate $E$. coli pan-genome, based on 24 E. coli and 8 Shigella genomes available at the time of analysis (November 2006). The inclusion of Shigella genomes was justified as the genus division between Shigella and Escherichia is historical but taxonomically incorrect $[5,6]$. For simplicity, the Shigella and E. coli genomes are collectively referred to as $E$. coli. From these genomes we construct an E. coli pan-genome microarray. The technical performance of this pan-genome microarray is assessed by the correct identification of present and absent genes from the completely sequenced genome of the MG1655 isolate of $E$. coli strain K-12 (hereafter referred to as MG1655) and strain O157:H7 EDL933 (EDL933 for short), collectively referred to as the control strains. Pathogenic E. coli isolates are highly overrepresented in the available genome sequences and, hence, on our pan-genome chip. We assessed whether this chip could nevertheless be useful for characterization of nonpathogenic isolates by hybridizing four probiotic $E$. coli isolates to the chip. These isolates are part of a commercially available product (Symbioflor2) marketed for human use as an enhancer of the immune system. The product contains viable bacteria comprising at least four genotypes of commensal E. coli. By characterizing their gene content, we investigated the phylogenetic relationship of these isolates to other E. coli strains.

\section{Results}

\section{Defining the $E$. coli core-genome and pan-genome}

For each of the considered genome and plasmid sequences listed in Table 1, genes were predicted by EasyGene $[7,8]$ and translated into proteins. These were considered conserved (belonging to the same protein gene group) if they showed a sequence similarity of $50 \%$ or higher along at least $50 \%$ of the full length of the protein sequence according to the similarity criteria defined in [3] (see Materials and methods for details). The core genome, that is, the number of conserved genes present in all genomes, was estimated by fitting an exponential decay function by non-linear least squares (Figure 1). In short, for each number of genomes (n), the gene content was compared for multiple random combinations of $\mathrm{n}$ genomes after which a best fit decay curve was fitted. Two slightly different decay functions were used: the originally suggested decay function based on [3] (Figure 1, green line) did not fit the data as well as a slightly modified exponential decay function (Figure 1, red line) (see Materials and methods for details on the applied modifications). Based on the best-fitting extrapolation, we estimate the size of the core genome to approach approximately 1,563 genes for an infinite (or very large) number of $E$. coli genomes.

We next estimated how many additional 'strain-specific' genes would be added to the core genome with each genome being sequenced. In this case the decay function defined by [3] was found to be appropriate, as shown in Figure 2. By fitting the data to the number of sequenced genomes approaching infinity, we predict that additional genomes will continue to add approximately 79 genes to the $E$. coli pan-genome, on average. Exploiting the fitted parameters for the data set, the size of the current $E$. coli core genome conserved within the 32 strains included in this study was estimated to contain 2,241 common genes. The estimated size of the current pangenome was estimated to contain 9,433 different genes. The number of $E$. coli strains used for these estimates is approxi- 
Table I

\begin{tabular}{|c|c|c|c|c|c|}
\hline Strain & Accession & NCBI Proj ID & Contigs & ORFs & Length \\
\hline E. coli 042 chromosome & $-*$ & 340 & 1 & 4,607 & $5,241,977$ \\
\hline E. coli 042 plasmid & - & 340 & 1 & 106 & 113,346 \\
\hline E. coli I0I-I chromosome & AAMK0I00000I-70 & 16193 & 70 & 4,353 & $4,880,382$ \\
\hline E. coli 53638 chromosome & AAKBOI00000I-119 & 15639 & 119 & 4,779 & $5,289,471$ \\
\hline E. coli 536 chromosome & СР000247 & 16235 & 1 & $4,34 I$ & $4,938,920$ \\
\hline E. coli B chromosome & - & 18083 & 1 & 4,076 & $4,629,819$ \\
\hline E. coli BI7I chromosome & AAJX0100000I-159 & 15630 & 159 & 4,780 & $5,299,753$ \\
\hline E. coli BI7I plasmid & AB024946 & 15630 & 1 & 69 & 68,817 \\
\hline E. coli B7A chromosome & AAJT0100000I-I98 & 15572 & 198 & 4,646 & $5,202,558$ \\
\hline E. coli CFT073 chromosome & AEOI4075 & 313 & 1 & 4,653 & $5,231,428$ \\
\hline E. coli EII0I9 chromosome & AAJWOI00000I-I5 & 15578 & 115 & 4,839 & $5,384,084$ \\
\hline E. coli E22 chromosome & AAJV0100000I-109 & 74230453 & 109 & 4,943 & $5,516,160$ \\
\hline E. coli E2348 chromosome & - & 341 & 4 & 4,592 & $5,071,653$ \\
\hline E. coli E2348 pB I7I plasmid & - & 341 & 1 & 70 & 68,890 \\
\hline E. coli E2348 p9123 plasmid & - & 341 & I & 5 & 6,293 \\
\hline E. coli E2348 pGEPAT plasmid & - & 341 & 1 & 3 & 2,233 \\
\hline E. coli E24377A chromosome & AAJZ0I00000I & 13960 & I & 4,407 & $4,980,187$ \\
\hline E. coli FII chromosome & AAJU0I00000I-88 & 15576 & 88 & 4,593 & $5,206,906$ \\
\hline E. coli HIO407 chromosome & - & - & 89 & 4,865 & $5,428,706$ \\
\hline E. coli HS chromosome & AAJYOI00000I & 13959 & 1 & 4,126 & $4,643,538$ \\
\hline E. coli KI2-MGI655 chromosome & U00096 & 225 & 1 & 4,122 & $4,639,675$ \\
\hline E. coli KI2-W3IIO chromosome & AP009048 & 16351 & 1 & 4,133 & $4,646,332$ \\
\hline E. coli OI03Oslo chromosome ${ }^{\dagger}$ & - & - & 1115 & 4,571 & $5,231,845$ \\
\hline E. coli OI57RIMD0509952 chromosome & BA000007 & 226 & 1 & $4,989 \ddagger$ & $5,498,450$ \\
\hline E. coli OI57RIMD0509952 pOI57 & $\mathrm{AB} 011549$ & 226 & 1 & 70 & 92,721 \\
\hline E. coli OI57RIMD0509952 pOSAKI & AB0II548 & 226 & 1 & 3 & 3,306 \\
\hline E. coli RS2 8 chromosome & - & - & 1 & 4,898 & $5,089,234$ \\
\hline E. coli RS2I 8 plasmid & - & - & 1 & 115 & 114,233 \\
\hline E. coli UTII 89 chromosome & СР000243 & 16259 & 1 & 4,466 & $5,065,741$ \\
\hline E. coli UTII 89 plasmid & СР000244 & 16259 & 1 & 114 & 114,230 \\
\hline E. coli VR50 chromosome ${ }^{\dagger}$ & - & - & 1228 & 4,453 & $5,064,870$ \\
\hline E. coli APEC-OI chromosome & СР000468 & 16718 & 1 & 4551 & $5,082,025$ \\
\hline E. coli OI 57EDL933 chromosome & NC_002655 & 259 & 1 & $4,664 \ddagger$ & $5,528,445$ \\
\hline E. coli OI57EDL933 plasmid & AF0746I3 & 259 & 1 & 70 & 92,077 \\
\hline S. boydii Sb227 chromosome & СР000036 & 13146 & I & 4,356 & $4,519,823$ \\
\hline S. dysenteriae MI 31649 chromosome & - & 346 & 234 & 4,755 & $4,962,690$ \\
\hline S. dysenteriae SdI 97 chromosome & СР000034 & 13145 & 1 & 4,237 & $4,369,232$ \\
\hline S. dysenteriae Sd I 97 pSDII 97 & СР000035 & 13145 & 1 & 160 & 182,726 \\
\hline S. flexneri $2457 \mathrm{~T}$ chromosome & AEOI 4073 & 408 & 1 & 4,388 & $4,599,354$ \\
\hline S. flexneri 301 chromosome & AE005674 & 310 & 1 & 4,410 & $4,607,203$ \\
\hline S. flexneri $30 \mathrm{I}$ pCP30I plasmid & AF386526 & 310 & 1 & 194 & 221,618 \\
\hline S. flexneri 8401 chromosome & СР000266 & 166375 & 1 & 4,383 & $4,574,284$ \\
\hline S. sonnei $53 \mathrm{G}$ chromosome & - & - & 5 & 4,780 & $5,220,473$ \\
\hline S. sonnei Ss 046 chromosome & СР000038 & $1315 \mid$ & I & 4,443 & $4,825,265$ \\
\hline S. sonnei Ss046 pSS plasmid & СР000039 & $13|5|$ & 1 & 179 & 214,396 \\
\hline
\end{tabular}

*In progress: the genome sequence has not been fully completed and an accession number has not yet been assigned.

tSequences generated using 454 technology representing a large number of contigs. These are almost certainly not complete.

‡These genes were predicted using EasyGene version I.2. All other genes were predicted using EasyGene version I.0. 


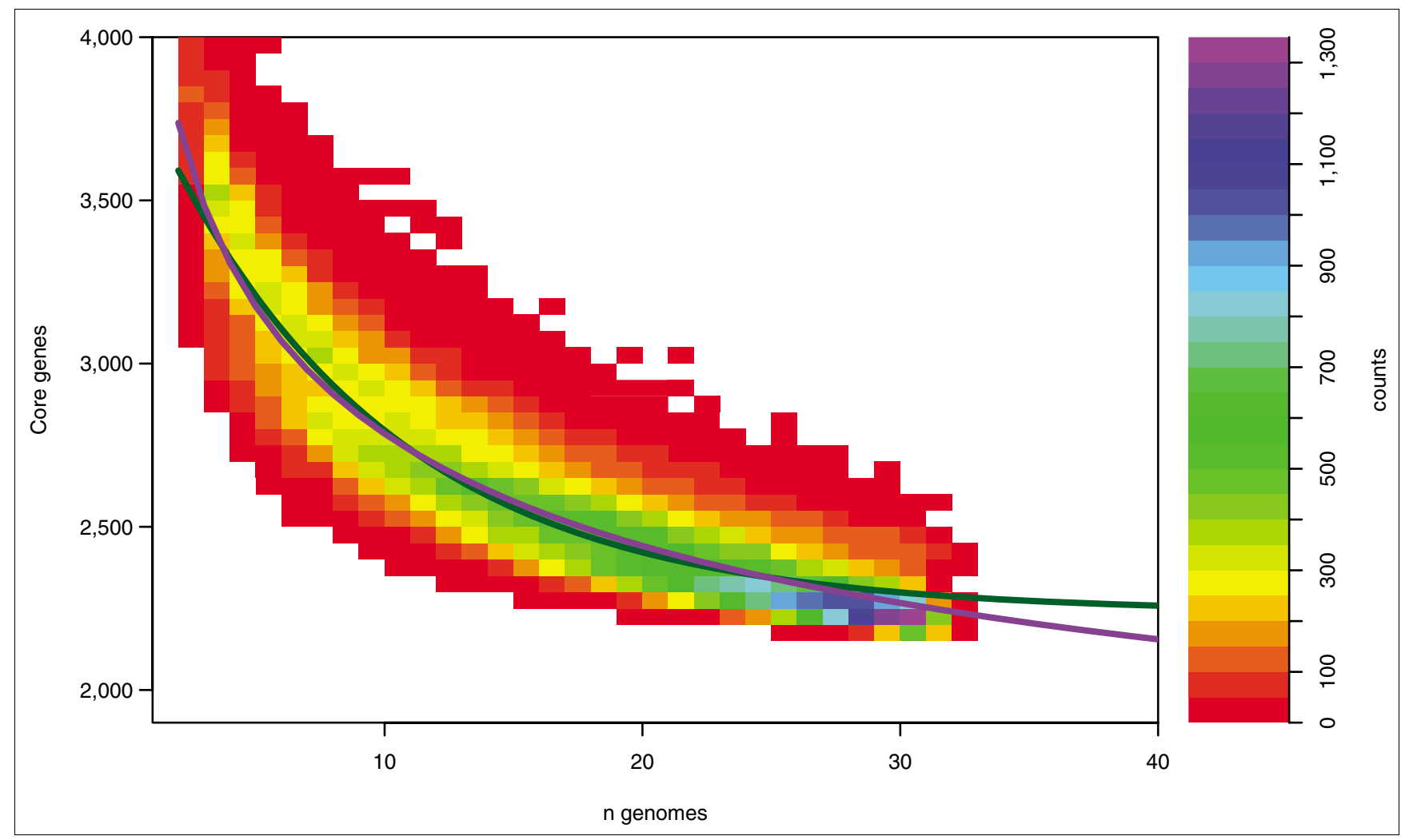

Figure I

Two-dimensional density plot of 'core genes' for the $E$. coli pan-genome. The plot illustrates the number of $E$. coli core genes for $n=2, \ldots, 32$ genomes based on a maximum of 3,200 random combinations of genomes for each $n$. The density colors reflect the count of combinations giving rise to a certain number of core genes; that is, for $n=3$, genome number 3 is compared to genomes I and 2, and the number of core genes is the number of genome 3 genes conserved in genomes I and 2 . The green line is the fit to the exponential decay function by [3], and the red line is our proposed fit to a slightly modified decay function as explained in the Materials and methods.

mately the same as the number of strains present in the human gut [9,10]; thus, the number of $E$. coli genes in the human gut is roughly a third of the number of human genes.

In designing the $E$. coli pan-genome microarray, genes were grouped based on their nucleotide sequences since the probes are based on DNA oligonucleotides. Moreover, the parameters to group genes for similarity were adapted compared to the parameters used for protein similarity to define the core and pan-genome in order to improve differentiation between the nucleotide sequences of similar $E$. coli genes found in different strains. For this purposes the ' $50 \%$ sequence similarity of $50 \%$ of the sequence' conservation criteria [3] was found to be sub-optimal. Instead, genes were grouped into gene groups with a slightly different and somewhat stricter homology criteria (see Materials and methods for details), producing a higher number of groupings. This resulted in a total of 11,872 gene groups present in all 32 genomes, compared to the smaller pan-genome of 9,433 gene groups resulting from comparison at the protein sequence level. Of the 11,872 gene groups, 2,041 consisted of genes found in all 32 strains. Thus, the stricter grouping criteria applied here produced a lower number than the currently estimated core genome size of 2,241 protein gene groups for $32 \mathrm{E}$. coli genomes.

In the presented design strategy, the inclusion of $32 \mathrm{E}$. coli strains in the microarray design necessitated the employment of a common standardized gene prediction strategy since some of the genomic sequences had poor or non-existing gene annotations. One option is to either include as many open reading frames as possible as potential genes (in a 'more is better' strategy) or, alternatively, to use EasyGene, a well performing and conservative gene predictor. One can argue that a 'more is better' strategy is preferred to the conservative gene prediction so that fewer genes would be missed. However, including spurious hypothetical genes in the design would potentially obstruct the probe design phase both in the grouping of gene families and in excluding otherwise perfect probes due to cross-hybridization to these false genes. Furthermore, in case of prediction of gene content in control and novel strains by hybridizing genomic DNA to the array, such false positives are just as unwelcome as false negatives. Nonetheless, absence of too many important $E$. coli genes is not desirable either. We therefore compared the genes predicted by 


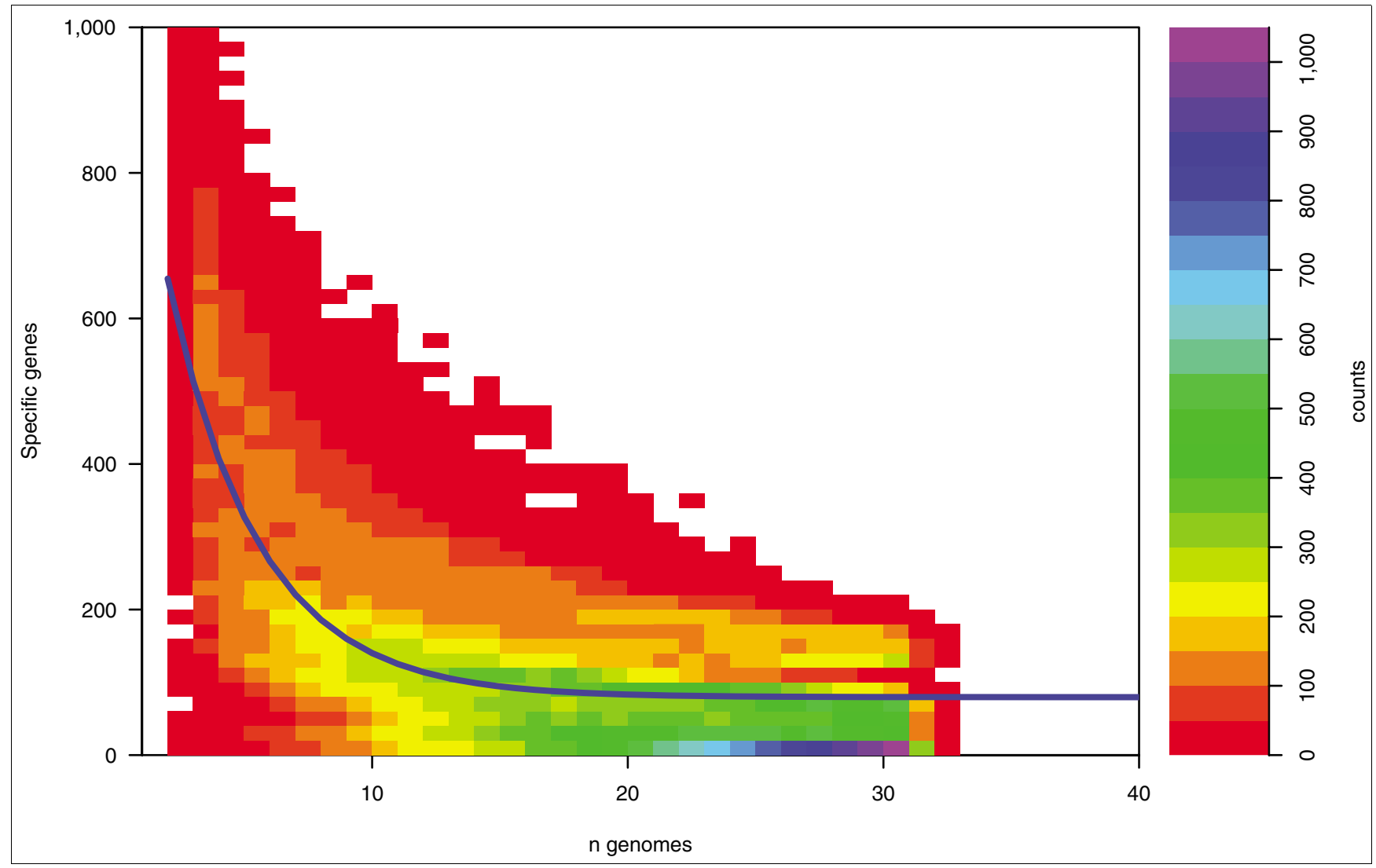

\section{Figure 2}

Two-dimensional density plot of novel genome 'specific genes' for the $E$. coli pan-genome. The plot illustrates the number of novel genome specific genes for the $n$th genome when comparing $n=2, \ldots, 32$ genomes (for a maximum of 3,200 random combinations at each $n$ ). The density colors reflect the count of combinations giving rise to a certain number of specific genes ( $y$-axis) in one genome compared to $n-I$ other genomes; that is, for $n=2$, genome number 2 is compared to genome number I and, on average, approximately 650 genes are found to be specific to strain 2 . The blue line is the fit to the originally suggested exponential decay function [3].

EasyGene with the high-quality annotation of the K-12 MG1655 strain (version Uooo96.3). This revealed that of the 238 protein encoding genes not predicted by EasyGene, 206 were hypothetical genes, leader peptides, frameshifts, gene fragments or pseudogenes. Of the remaining 32 genes, 12 were present in at least one other $E$. coli strain considered in the design. Consequently, only 20 genes of potential interest were missed by EasyGene. Since this is less than half a percent of the genome $(20 / 4,331=0.46 \%)$, we considered that the advantages of conservative standardized gene finding outweighed the disadvantages of missing a small minority of genes.

\section{Benchmarking the chip design}

A pan-genomic approach represents a challenge in evaluating and defining the trade-off in group inclusion stringency: a similarity cut-off chosen too high will result in too many groups, while a low similarity cut-off results in too much sequence variability within a group (producing low conservation scores). Consequently, too much sequence variability within groups will result in group-specific probes producing too low a signal for that group in particular strains. On the other hand, dividing the groups further to limit this undesired inter-group variability causes another problem: some probes may no longer be group specific, leading to undesired crosshybridization, while other probes might still provide a signal specific for such a group. In the attempt to circumvent these problems, an additional filter step was introduced in the probe design strategy, where probes were removed from further analysis if they were not specific enough to one group and if they did not share a sequence overlap above a certain threshold with the sequences in the group it was designed for (for details refer to Materials and methods). Figure 3a gives an example of how such probes may result in misleading signals, while the signal improves remarkably following exclusion of such probes from the analysis by a filtering step (Figure 3b).

The chip design was assessed by analyzing and comparing the hybridization data from the two sequenced control strains, EDL933 and MG1655. Both $\log _{2}$ intensities and $\log _{2}$ ratios were considered. These results are visualized in a hybridization atlas (Figure 4). Here, the median $\log _{2}$ intensity and $\log _{2}$ ratios of both control strains are illustrated for MG1655 


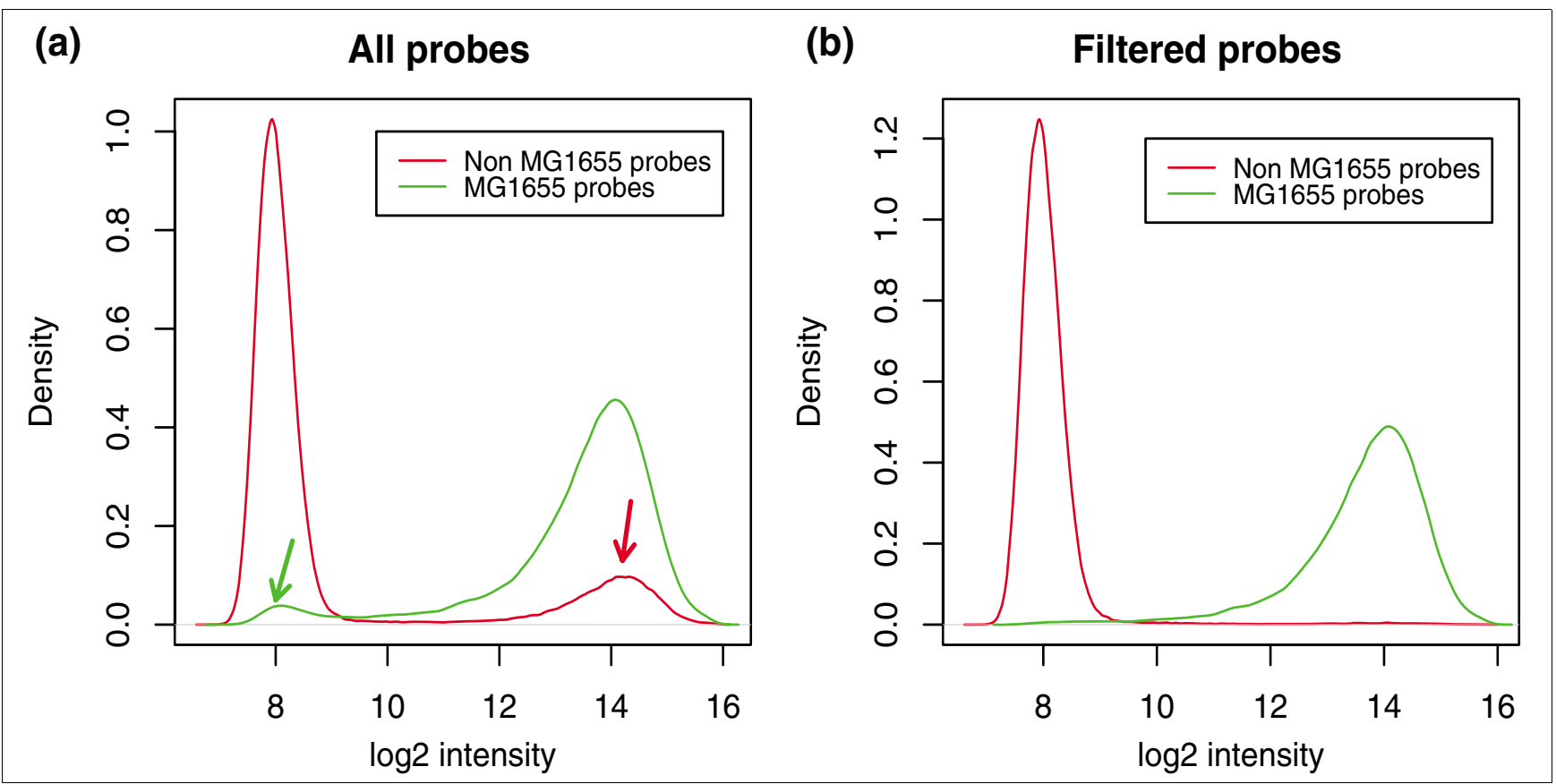

Figure 3

Density plots of probe intensities before and after a filtering step. The density distributions are illustrated for MGI655 probes and non-MGI655 probes separately. Log $_{2}$ intensity data are from a representative MGI655 control sample. (a) Before filtering, all probes are divided into MGI655 probes (green lines) and non-MG 1655 probes (red lines). It is clear that many probes initially designed for groups containing MGI655 genes do not hybridize well to these, resulting in low intensity (green arrow). Conversely, probes initially designed for groups without MGI655 genes cross-hybridize as if present in MGI655 (red arrow). (b) After filtering probes, the remaining probes have the expected hybridization profile.

probes, as well as probe coverage for this strain and the sequence similarity at the DNA level of EDL933 genes to MG1655 genes based on BLAST scores. The similarity of the MG1655 probe hybridization pattern for EDL933 to the sequence similarity based on BLAST scores confirms that the probes reflect true biology. The same information is illustrated in the ratio circle (fourth outermost circle), where MG1655 regions absent in the EDL933 genome are clearly visible and correspond to the regions missing in the EDL933 sample (first and second outermost circle). On the contrary, the MG1655 hybridization pattern (third outermost circle) corresponds very well to the probe coverage pattern (innermost circle).

For further analysis, the probes were mapped to each gene group according to the design, and a position-dependent segmentation algorithm was employed to partition data points into present and absent sequence segments [11]. Segmentation was followed by merging the output with MergeLevels [12]. Since the distribution of $\log _{2}$ intensities is bimodal - that is, composed of two density distributions (Figure 5a) - it is likely that the best separation of present and absent probes can be found at the local minimum between the two distributions. Consequently, following noise reduction by segmentation and merging, the cutoff for $\log _{2}$ intensities was found at the merged value between these two distribution maxima with the least segments assigned to it. All segments with merged values above this cutoff were predicted as present. On the other hand, the distribution of $\log _{2}$ ratios is largely unimodal (although two extra, weaker modals occur) (Figure $5 \mathrm{~b}$ ). Since ratios are only calculated for genes present in the control sample, and given the likely high similarity between a test sample and control sample of the same species, most genes are assumed present. Consequently, here the present level was estimated as the merged level to which most segments had been assigned.

Following the filtering step, several gene groups were left with only few probes targeting them, and we found it necessary to remove groups that were targeted by three or fewer probes from further analysis. This reduced the average number of false positives from 267 to 87 (for MG1655) and from 638 to 405 when analyzing all control samples with regard to genes found to be present from analysis of $\log _{2}$ hybridization signals compared to genes predicted present from the genome sequence. On the other hand, gene groups represented by few probes were not as likely to result in false negatives since removal of these groups did not change the average number of false negatives significantly (data not shown).

Table 2 lists the resulting sensitivity and false discovery rate (FDR) for all control samples. A very high sensitivity was obtained for both strains, but false positives were suspiciously high for EDL933 (Table 2). For both control strains, a large 


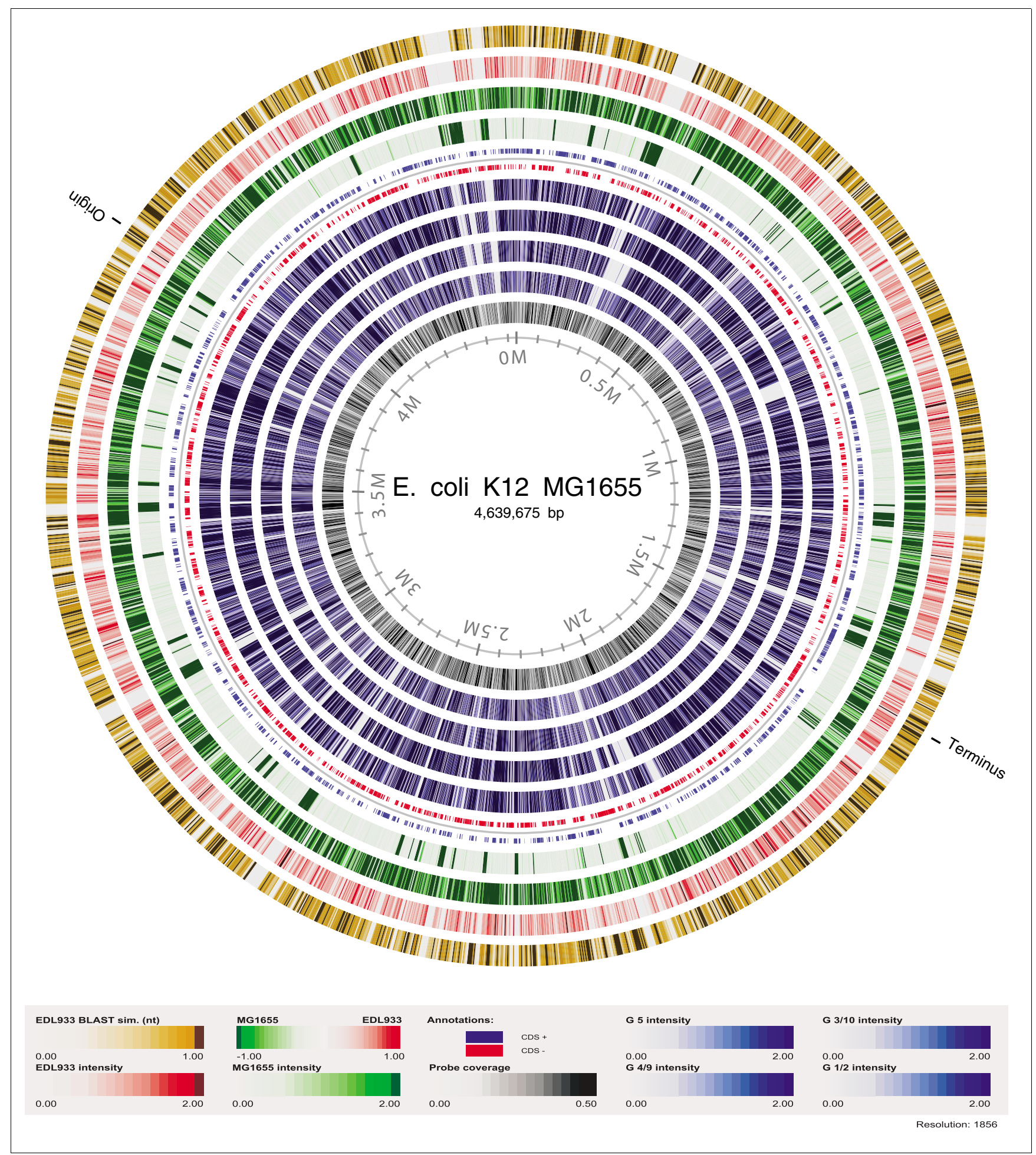

Figure 4

Hybridization and blast atlas. The atlas illustrates the hybridization pattern of MGI655 probes for the two control strains, MGI655 and EDL933, and the four Symbioflor2 isolates. Also, it illustrates the MG 1655 genes' BLAST score for presence in the EDL933 strain. The circles from outermost to innermost are: Blast score between 0 for absent and I for present MG 1655 genes in the EDL933 strain, $\log _{2}$ transformed hybridization intensities for EDL933 and MG 1655 samples, $\log _{2}$ ratio of EDL933/MGI 655 samples, location of predicted coding sequences (CDS), $\log _{2}$ hybridization intensities for the four Symbioflor2 isolates $\mathrm{G} 5, \mathrm{G} 4 / 9, \mathrm{G} 3 / \mathrm{I} 0, \mathrm{GI} / 2$, probe coverage. A zoomable version of the atlas is available at [33]. 


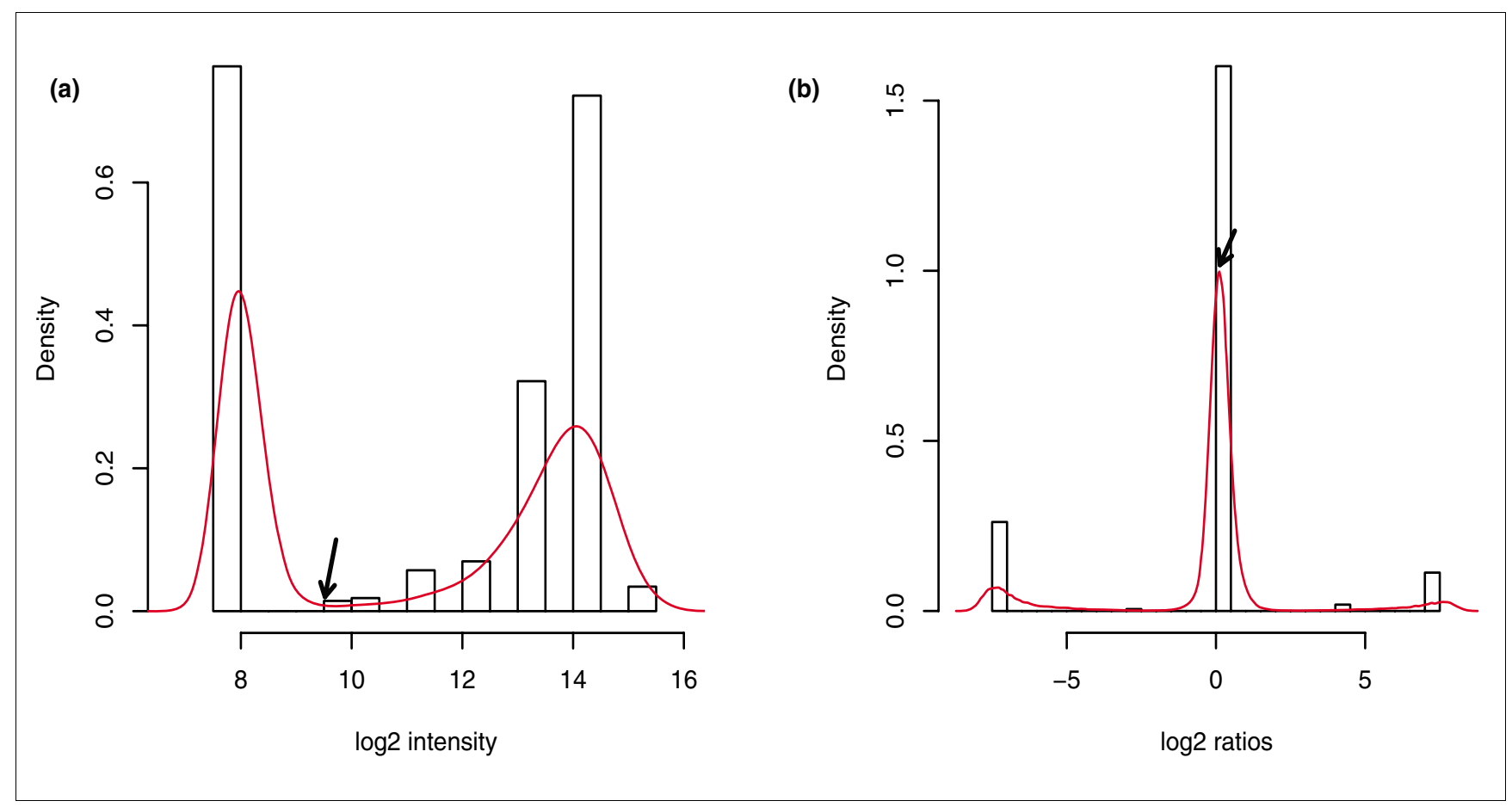

Figure 5

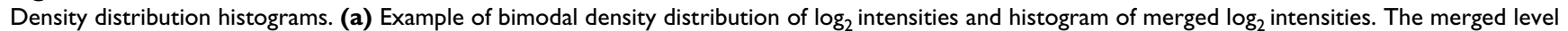
with fewest segments assigned to it is chosen as the cutoff value. All segments with merged values above this cutoff are predicted as present. An arrow indicates the cutoff level for this particular sample. (b) Example of unimodal (or trimodal) density distribution of $\log _{2}$ ratios and histogram of merged ratios. The merged level with the most segments assigned to it was chosen as the present level. All segments with this merged value or above were predicted as present. An arrow indicates the minimum $\log _{2}$ ratio for present probes for this particular sample.

proportion of the false positive gene groups were consistently identified in replicate samples (a total of 62 and 360 in MG1655 and EDL933, respectively). For MG1655, genes annotated as hypothetical were highly overrepresented among the false positive genes ( $P$ value approximately 0.002 , Fischer's exact test), indicating a significant enrichment in hypothetical genes among false positives. In the majority of cases, the corresponding consensus sequences aligned very well to the genome sequence (with $>50 \%$ of the sequence length and $>91 \%$ identity). Consequently, these false positives are not a result of cross-hybridizations but rather a result of genes not predicted by the EasyGene gene finder. Since most of these are seemingly hypothetical and, therefore, are likely not to be real genes, the consequences in terms of strain characterization are considered to be minor.

Table 2

Sensitivity and false discovery rate based on analysis of $\log _{2}$ intensities

\begin{tabular}{|c|c|c|c|c|c|}
\hline \multicolumn{3}{|c|}{ MGI655 } & \multicolumn{3}{|c|}{ EDL933 } \\
\hline Chip ID & Sensitivity & FDR & Chip ID & Sensitivity & FDR \\
\hline 108276 & 0.988 & 0.021 & 1004602 & 0.994 & 0.13 \\
\hline 108667 & 0.964 & 0.024 & 113504 & 0.988 & 0.12 \\
\hline 113756 & 0.997 & 0.021 & 113509 & 0.980 & 0.12 \\
\hline 114782 & 0.999 & 0.017 & II 3757 & 0.989 & 0.13 \\
\hline 1509502 & 0.999 & 0.043 & 1509502 & 0.970 & 0.11 \\
\hline 1510802 & 0.999 & 0.015 & 1510802 & 0.994 & 0.11 \\
\hline Average & 0.989 & 0.024 & Average & 0.986 & 0.12 \\
\hline
\end{tabular}

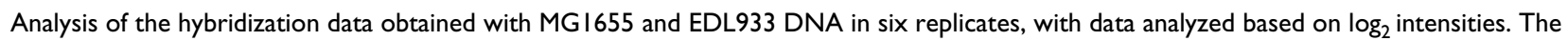
sensitivity and false discovery rate (FDR) are given for the prediction of gene presence in MGI655 or EDL933 in the corresponding samples. 
In contrast to the MG1655 control strain, we did not observe enrichment in hypothetical genes among false positives for EDL933. In this case we suspect that the 'false positives' were actually true genes mistakenly missed by EasyGene. In support of this, EasyGene did actually predict only 4,664 genes for the EDL933 main chromosome compared to the 5,349 annotated in GenBank, possibly due to a number of unknown nucleotides still present in the published genome sequence [13]. Gene expression profiling of these genes would confirm if these are in fact true genes that are expressed and thus incorrectly missed by EasyGene. Preliminary data from a gene expression study run in parallel with this work demonstrated that the gene expression profile of these genes indeed resembled that of other genes present in the EDL933 genome (Sekse C, Friis C, Wasteson Y, Ussery DW and Willenbrock $\mathrm{H}$, unpublished results). This observation supports our interpretation that they are actually not false positives generated by bad chip manufacturing, hybridization artifacts or poor analysis approaches, but a consequence of an ambiguous DNA sequence that any gene predictor would have ignored. Ideally, they should have been categorized as true positives. Consequently, the low FDR obtained from the other control strain, MG1655, is a better indicator of our pan-genome chip performance.

Table 3 compares the performance obtained by analyzing $\log _{2}$ ratios of control sample co-hybridizations with the performance based on $\log _{2}$ intensities. In both cases, the sensitivity is quite high, while FDR is low, in particular for MG1655. The higher FDR for EDL933 may be assigned to a low accuracy for the gene predictor on this particular genome, as discussed above. While the sensitivity is slightly higher when analyzing $\log _{2}$ ratios, FDR is marginally lower when analyzing $\log _{2}$ intensities. Consequently, the single channel $\log _{2}$ intensity analysis approach offers an acceptable performance compared to the comparative dual channel approach, at a limited risk of increased false negatives but with the added advantage of being able to identify the presence and absence of any gene on the microarray and not only genes present in the control strain.

\section{Analysis of probiotic $E$. coli strains}

The chip design was next tested for suitability to characterize isolates of non-pathogenic $E$. coli strains. Four probiotic isolates were co-hybridized with MG1655 and EDL933 according to the combinations listed in Table 4; their hybridization pattern to MG1655 probes is illustrated in a hybridization atlas (Figure 4). Here, larger regions absent from the probiotic isolates in comparison to MG1655 are visible. It is also evident that each isolate is different from the next, since each isolate has a distinct hybridization pattern.

The gene content of each probiotic isolate was predicted by the single-channel approach as found to be appropriate for this type of analysis. Thereby, the presence of all genes included on the pan-genome array could be assessed for all four test isolates. First, we compared the findings between the isolates used for hybridization. The number of identified genes was highest for G1/2 and lowest for G4/9 (Table 5). Two graphical representations further illustrate the results. Figure 6 shows a cluster analysis based on all probes considered in this paper. The four probiotic isolates cluster individually and form a super-cluster with MG1655 samples, separated from EDL933. Indeed, each isolate shared more of their predicted genes with MG1665 than with EDL933 (Table 5). Moreover, strain-specific genes were more frequently different to EDL933 than to MG1655. This is not surprising since the probiotic isolates are likely to be more related to the non-pathogenic commensal K-12 than to enterohemorrhagic EDL933. Each strain had more than 100 genes that were neither found in MG1655 nor EDL933 (Table 5). Moreover, a significant enrichment was observed in hypothetical genes among the gene groups only found in a single Symbioflor2 isolate. However, this is expected, since $E$. coli core genes are generally better characterized than genes found in only few $E$. coli strains. Figure 7 compares the numbers of genes found to be either unique or shared between one or more probiotic isolates in a Venn diagram. A total of 3,093 genes were found consistently in all four isolates. Figure 6 and Figure 7 both identify isolate G1/2 as the most distantly related to the other isolates.

Table 3

$\log _{2}$ intensity results versus $\log _{2}$ ratio results for test samples MG 1655 and EDL933

\begin{tabular}{|c|c|c|c|c|}
\hline & \multicolumn{2}{|c|}{$\log _{2}$ intensities } & \multicolumn{2}{|c|}{$\log _{2}$ ratios } \\
\hline & MGI655 & EDL933 & MGI655 & EDL933 \\
\hline Sensitivity & 0.99 & 0.97 & 1.00 & 1.00 \\
\hline FDR & 0.003 & 0.060 & 0.007 & 0.063 \\
\hline
\end{tabular}

The sensitivity and false discovery rate (FDR) were compared for data analysis based on $\log _{2}$ intensities and $\log _{2}$ ratios for the detection of genes in the two control strains for which gene presence is known from gene finding based on the known genome sequence. Thus, only known control gene groups were considered. Consequently, true positives make up the control genes correctly found to be present in all MGI655 or EDL933 samples, respectively. False positives are genes not found in the control strain, but predicted as present from the genome sequence. 
Table 4

\begin{tabular}{lll}
\hline Co-hybridization setup & & \\
\hline Chip ID & Cy3 (test) & Cy5 (control) \\
\hline 113756 & G I/2 & MGI655 \\
108667 & G 3/I0 & MGI655 \\
114782 & G 4/9 & MGI655 \\
108276 & G5 & MG I655 \\
113509 & G I/2 & EDL933 \\
113504 & G 3/10 & EDL933 \\
113757 & G5 & EDL933 \\
1004602 & G 4/9 & EDL933 \\
1509502 & EDL933 & MG I655 \\
1510802 & EDL933 & MG I655 \\
\hline
\end{tabular}

Next, genes detected in the probiotic isolates were compared to the genes present (by gene prediction based on their genome sequence) in each $E$. coli strain represented by the chip. All four probiotic isolates shared the most genes with $E$. coli $\mathrm{H} 10407$, closely followed by the two K-12 strains for three of the isolates and the VR50 strain for G1/2 (refer to Table S1 in Additional data file 1 for a ranked list of the number of shared genes with the strains considered for chip design). While E. coli VR50 is an asymptomatic inhabitant of the urinary tract [14], E. coli $\mathrm{H} 10407$ is an enterotoxigenic strain. However, its virulence is mostly encoded by plasmids that have not yet been sequenced and, therefore, were not considered in this comparison. Nonetheless, by gene prediction based on the genomic sequence of the H10407 main chromosome, we identified the presence of genes encoding hemolysin (hlyCABD). These genes were present in probiotic isolate G1/2 as well, in accordance with its weak hemolytic phenotype (described as alpha hemolysis type II; L Beutin and K Zimmermann, unpublished results). Presence of this gene cluster is, however, not sufficient to characterize an isolate as pathogenic [15-17]. Also, the main chromosome of the $\mathrm{H} 10407$ strain has previously been found to be highly homologous to $E$. coli K-12 in contrast to other $E$. coli pathogenic strains [18]. This indicates that in spite of the many genes shared with a pathogenic $E$. coli strain, the probiotic isolates are likely to share only the non-virulent parts. Besides, the probiotic isolate shares only marginally more genes with the $\mathrm{H} 10407$ strain than with the two K-12 strains (16-57 genes). This is not significant, especially since novel strains are much more likely to share more genes with the large H10407 genome than with the smaller K-12 genomes without actually resembling it more, simply because the H10407 genome encodes 20\% more genes. Supporting this, a cluster analysis considering the presence and absence of all gene groups analyzed from our pan-genome array (Figure 8) clearly shows that the gene content of the probiotic isolates is, in fact, more closely related to the gene content of other nonpathogenic strains. In this analysis, all probiotic isolates cluster together with the two K-12 strains while forming a supercluster with all the other non-pathogenic strains considered

Table 5

Comparison of Symbioflor2 isolates to predictions for control strain samples

\begin{tabular}{|c|c|c|c|c|}
\hline & $\mathrm{G} I / 2$ & G 3/10 & G 4/9 & G5 \\
\hline No. of predicted genes & 3,978 & 3,683 & 3,568 & 3,660 \\
\hline \multicolumn{5}{|c|}{ No. of genes in common with (based on $\log _{2}$ intensities): } \\
\hline MGI655 & 3,464 & 3,323 & 3,319 & 3,399 \\
\hline EDL933 & 3,455 & 3,264 & 3,186 & 3,237 \\
\hline \multicolumn{5}{|c|}{ 'Novel' sample genes not in (based on $\log _{2}$ intensities): } \\
\hline MGI655 & 358 & 251 & 162 & 197 \\
\hline EDL933 & 631 & 647 & 635 & 592 \\
\hline Either control & 185 & 197 & 126 & 144 \\
\hline
\end{tabular}

Results are based on $\log _{2}$ intensity analyses. 


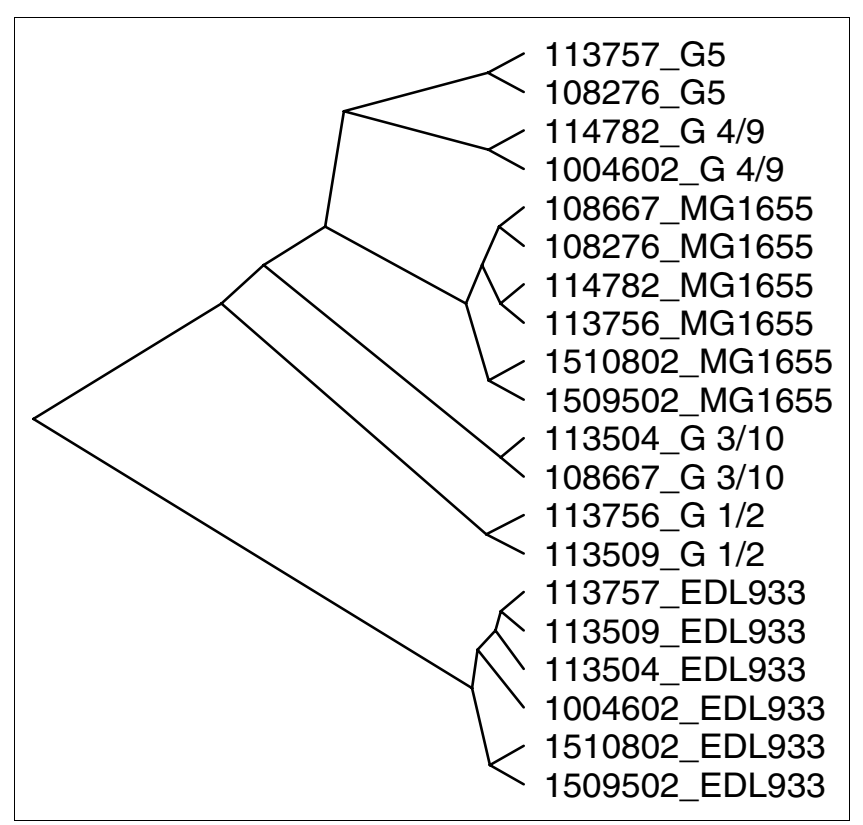

Figure 6

Hierarchical cluster analysis of hybridization signals from the samples summarized in Table 4, including control samples. The analysis is based on remaining probes (refer to Materials and methods for details) after filtering and removal of probes in gene groups with three or less probes. For clustering, the 'I-pearson correlation' distance metric was used.

in the analysis. This super-cluster contains only a few pathogenic strains for which the sequences of their virulence plasmids were not included in the analysis (strains 101-1, E24377A, and H10407). Furthermore, the validity of the clustering is confirmed by the placement of the control strain MG1655 closest to the two K-12 design strains, and EDL933 closest to the $\mathrm{O} 157: \mathrm{H} 7$ design strains.

Apart from the hemolysin genes and a gene annotated as 'putative iron-regulated outer membrane viruence gene', no other virulence genes were detected in the probiotic isolates. The observed genetic relatedness of probiotic strains to a virulent strain illustrates that both pathogenic and non-pathogenic $E$. coli strains use common strategies for adaptation to their niche. Of the genes found to be present in the probiotic isolates but not in a non-pathogenic E. coli strain (MG1655), many were bacteriophage-derived. Nevertheless, complete prophages were not present and variation between and within phage gene content between the four probiotic isolates suggested these bacteriophages have been introduced in independent events. Transposases and other insertion sequencerelated genes provided further evidence of the influence of mobile DNA on introducing genetic variation in a bacterial population. Of interest were genes present in the probiotic isolates but absent in MG1655 that were annotated as having general metabolic functions. A closer analysis of these findings would be necessary to assess if such genes provide improved fitness for colonization of the human gut, and so could explain the probiotic nature of the isolates. Also, one should keep in mind that the K-12 isolates represent a reduced $E$. coli genome, and some essential metabolic genes are known to be missing in these isolates. Complete lists of annotated genes found in each of the four Symbioflor2 isolates but not in the MG1655 control strain is provided as Additional data file 2.

\section{Discussion}

The design of a microarray covering more than 30 genomes proved to be a considerable challenge. Multiple aspects had to be considered but the greatest difficulty was to filter out false positives, at the risk of introducing additional false negatives. The level of similarity between gene sequences should justify conserved annotation, but the borders of significance are diffuse and poorly defined. This is a consequence of biological processes that undergo gradual genetic changes. On one hand, probes should cover all versions of the same gene, but at the same time they should be able to distinguish between different genes and even, when relevant, distinct versions of the same gene in different strains. In light of this, conventional microarray design strategies, such as inclusion of mismatch probes for background estimation, will not work when dealing with multiple genomes. One can never ensure that a perfect match is absent for such probes in novel strains. Moreover, because the array should be able to interrogate for the presence of genes at the DNA level (as presented in this paper), the number of probes per gene should be allowed to vary. A higher number of probes is required for a sufficient coverage of long genes, whereas low quality probes would result if attempting to design the same number of probes for very short genes. Consequently, the challenge is to define, in a sensible way, such goals and to search for the best possible solution. Our pan-genome approach proved to be a suitable solution.

Recently, the idea of an 'open pan-genome' was introduced, where each newly sequenced strain would continue to add novel genes to the pan-genome of the species. It was suggested that Streptococcus agalactiae would have an open pan-genome, with the consequence that despite sequencing hundreds of strains, novel genes would still be discovered [3]. $E$. coli is likely to also have an open pan-genome since it colonizes multiple environments, complex microflora biotopes, and, therefore, has multiple ways and sources for exchanging and obtaining genetic material [4]. In line with this expectation, Chen and co-workers [19] predicted that each new E. coli genome would add 441 genes to the $E$. coli pan-genome pool. However, this prediction of 'new genes' is possibly too high, since it was based on seven very diverse $E$. coli genomes only. Genome size differs considerably within the species, from the relatively small K-12 strains to the larger pathogenic $\mathrm{O}_{157}: \mathrm{H}_{7}$ strains. Therefore, we believe that our estimate of the $E$. coli pan-genome and the core genome is closer to what might be expected in the 'real world', since it is based on a much larger number and variety of strains. Thus, the number of added 


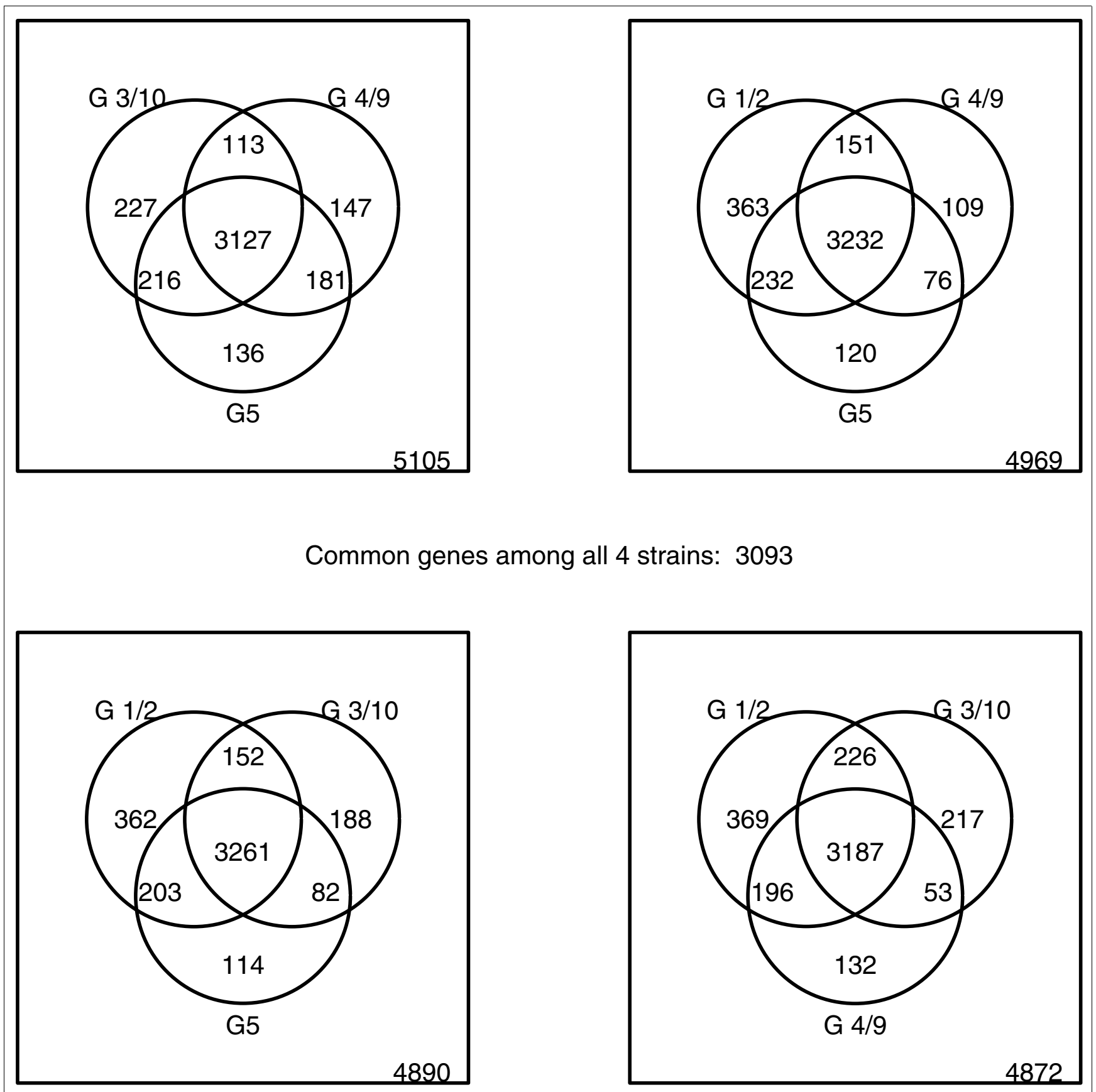

Figure 7

Venn diagram comparing Symbioflor2 isolates.

new genes per genome has dropped to about 79 genes when including data from 32 different strains, and may decrease further with improved genome annotations. This smaller estimate is in the same order of magnitude as predicted for other pan-genomes, such as Streptococcus (27 per genome for group A and 33 for group B) [3]. Still, our estimate for $E$. coli may be too conservative if the true diversity of $E$. coli is still insufficiently covered by the current genome sequences, that is, environmental and non-mammalian strains are under-represented, and the addition of these may initially add a significant number of novel genes to the $E$. coli pangenome.

Furthermore, we were able to come up with a more accurate prediction of the E. coli core genome. Previously, the size of the $E$. coli core genome was assessed, based on seven different $E$. coli strains, to consist of between 2,865 and 3,475 genes $[2,19]$. Based on the 32 genomes included in this study, we 


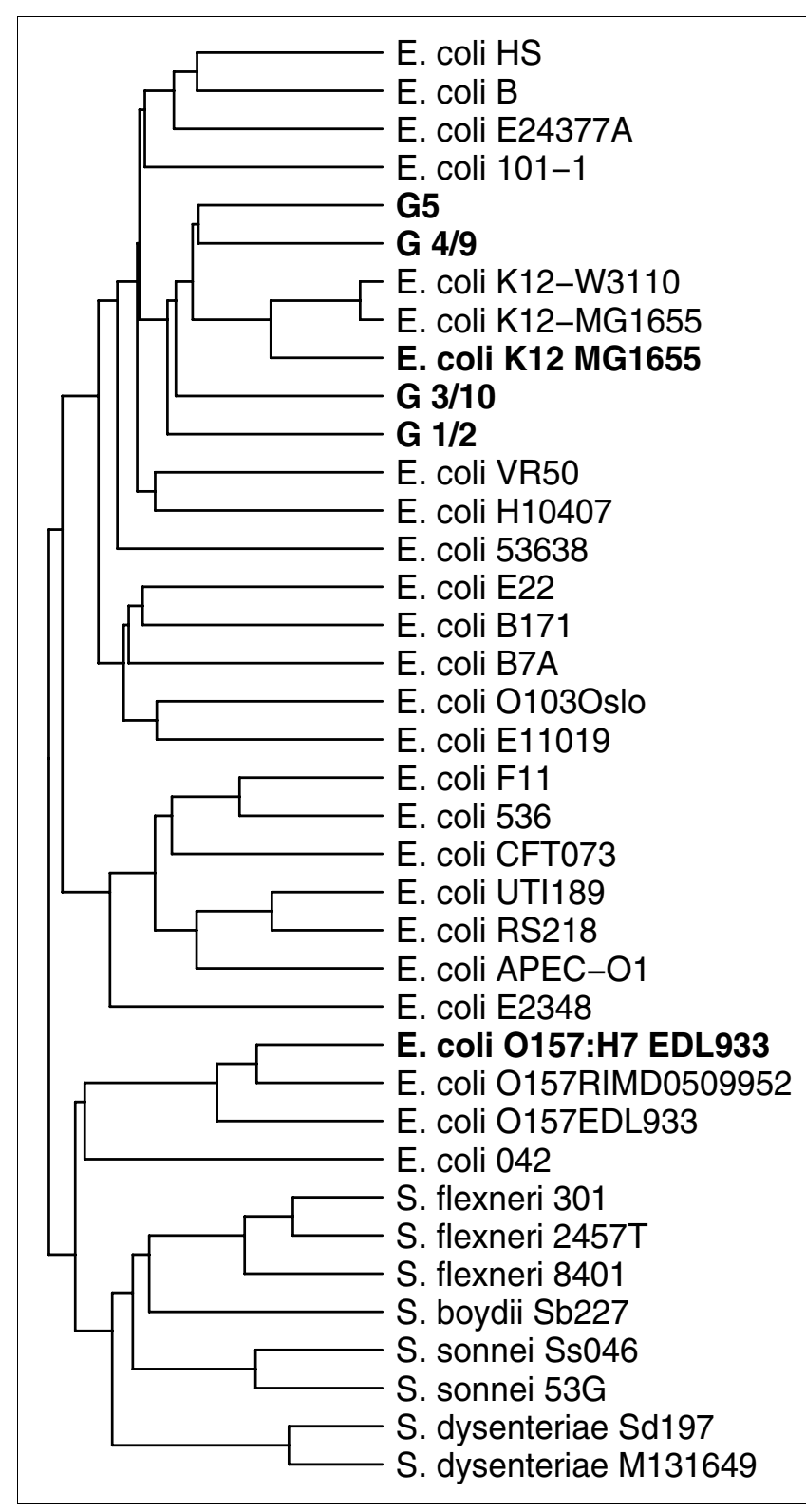

Figure 8

Hierarchical cluster analysis of the 32 design genomes according to gene absence or presence based on the design (normal font). The four Symbioflor2 isolates as well as the two control strains are included in the dendrogram (bold font) by clustering them according to gene predictions based on the sample hybridizations. For clustering, the Euclidian distance metric was used.

predict that the size of the core genome will approach approximately 1,560 essential genes, about half that of the previous estimates. We believe the current estimate to be more accurate, as it is based on a much larger number of genomes. However, in the present study, several unfinished genome sequences were included. Improving these both in terms of sequencing and assembly and in gene annotation quality, may result in an increased core genome size if the current partly finished genome sequences are missing core genes.
To assess the performance of the chip as well as to identify the best way of analyzing data from it, control sample hybridizations were analyzed. Comparative hybridizations on dual channel microarrays have the advantage of reduced noise due to limited variations of probe hybridization efficiencies. However, a dual channel analysis is limited to probes covering the control sample so that noise reduction applies only to probes hybridizing to genes present in the control sample. Although the false positive rate was slightly higher for the single-channel analysis approach, we demonstrate that sensitivity is only marginally lower than for the dual channel approach while information can also be extracted regarding genes not present in the control sample. Consequently, this analysis approach offers a favorable possibility for deriving predictions for any gene present on the pan-genome microarray.

Pathogenic E. coli genomes are highly overrepresented on this pan-genome chip because the majority of the $E$. coli genomes sequenced to date are from pathogenic strains, and few originate from environmental sources or are commensal strains. Nonetheless, we found that the chip was widely useful for characterizing the gene content of non-pathogenic $E$. coli isolates and for investigating the non-pathogenic nature of these $E$. coli isolates.

Lessons learned from this microarray can be used to design better arrays in the future. Although we considered all designed probes for the chip, including probes with low specificity to all strains in a given gene group, based on our analysis of experimental results, we have found that a filtering step is necessary to remove less specific probes. Moreover, gene groups for which only few probes could be designed (above the probe score cutoff) were not as reliable as gene groups represented by a larger number of probes. While this is not surprising, it nonetheless makes it a difficult task to accurately probe for these genes.

\section{Conclusion}

Based on 32 E. coli and Shigella genome sequences, we have developed an $E$. coli pan-genome microarray representing the current pan-genome of $E$. coli. Although any individual E. coli genome contains between 4,000 and 5,000 genes, we find about twice as many distinct gene groups in the total gene pool examined. High-density pan-genome microarrays can be quite useful for characterizing either DNA content or gene expression from unknown $E$. coli strains. Thus, we found the technique highly sufficient to investigate gene content of four non-pathogenic $E$. coli isolates despite the strong bias for pathogenic strains represented on the pan-genome array. The four analyzed probiotic $E$. coli isolates share a gene pool very similar to the $E$. coli K-12 strains, and additional strain-specific genes were often phage genes, transposases, insertion elements and metabolic genes. It remains to be seen to what degree these genes contribute to the probiotic nature of the isolates. Generally, we conclude that our high-density pan- 
genome array provides an excellent tool for characterizing the genetic makeup of unknown $E$. coli strains and can also deliver insights into phylogenetic relationships.

\section{Materials and methods}

Twenty-four E. coli chromosome sequences that were publicly available at the time of analysis (as one or multiple contigs) and nine plasmid sequences belonging to seven of the sequenced strains were included in this study. In addition, eight Shigella chromosomes were included (two S. sonnei, three $S$. flexneri, two $S$. dysenteriae and one $S$. boydii) with their three corresponding plasmids (Table 1).

\section{Probe and microarray design}

All considered genome and plasmid sequences (Table 1) were searched for genes using EasyGene version 1.0 or $1.2[7,8]$ in order to standardize gene finding. Genes were screened for homology using BLAST [20] in order to prevent redundancy of the probes. Genes were placed in a group when homologous by the following criteria: E-value $<10^{-5}$, bitscore $>55$, and alignment constituting 50\% or more of the longer of the two aligned sequences. Genes with no homology were represented as 'singles'. Groups of genes ('multiples') were aligned using ClustalW with default settings [21] and a consensus sequence was derived using the most frequent nucleotide at each position, weighted by its background frequency in all genes. The probe design strategy employed by OligoWiz [22] was used for probe selection. Two additional scores were introduced as parameters for the probe design software dealing with consensus sequences: a weighted conservation score and a gap score. The weighted conservation score uses Shannon's information measure [23] for conservation at each nucleotide position in a probe. According to [24], the influence of a mismatch on measured hybridization intensities varies with its position, with positions towards either end having less influence. Therefore, each position was weighted according to a second order polynomial function. The probe's weighted conservation score is the product of the weighted position scores for mismatch basepairs. The gap score was used to identify probes that targeted gaps in the alignment of multiples. This score was used to design probes that specifically identified conserved regions of all genes in each group (thus attempting to avoid gaps).

All probes were designed as 55-6o mers, with variable length and sequence to optimize for the same melting temperature. Only standard nucleotides (GATC) were considered in the probe design. In total, 305,285 probes covering 11,768 gene groups and singles were designed. A detailed description of the microarray design may be found in Additional data file 3 . The probe design was given the NimbleGen design_id 5137 and is available upon request.

\section{Filtering of probes}

Probes were aligned against all predicted gene sequences included using NCBI-BLAST blastn version 2.2.11 [25] and the identity of each probe with each gene sequence was determined in base pairs. Sequences were extracted for which the ratio [bp identity/probe length] was $>0.8$. Probes that either matched more than one single group or failed to match all genes in the design group were excluded from further analysis. Furthermore, groups for which three or less probes remained after filtering were removed from the subsequent analysis due to their increased risk of generating false positives (see Results). This resulted in a reduction to 224,805 probes covering 9,252 gene groups and singles. Consequently, the number of probes targeting each gene ranged from 4 to 29 with a median coverage of 27 probes per gene.

\section{Annotation of gene groups}

Each gene group in the probe design was annotated according to the results from hits against the UniProtKB/Swiss-Prot release 52.5 and UniProtKB/TrEMBL release 35.5 protein database [26] using NCBI-BLAST Blastp version 2.2.11 [25]. Only alignments covering $>50 \%$ of the gene lengths and having 50\% or better identity within the alignment were included. Among all the sequences within each group, the hit producing the highest percent identity was chosen. In this way, 5,348 of our 11,872 gene groups could be annotated against Swiss-Prot and 9,320 of our 11,872 gene groups could be annotated against TrEMBL. Thus, while Swiss-Prot generally produces more reliable annotations, the number of annotations produced was quite low. Consequently, when available, genes were assigned the more reliable Swiss-Prot annotation, otherwise it was assigned the TrEMBL annotation if one was available. Gene groups that could not be assigned an annotation were assigned hypothetical proteins.

\section{Pan-genomics}

The pan-genome was estimated as suggested by Tettelin et al. [3], with modifications to reduce computational load for our large dataset. Briefly, protein sequences were compared by Blastp version 2.2.11 [25]. Proteins with at least 50\% sequence identity over at least $50 \%$ of their length were identified as the same. For each $\mathrm{n}$ additional genome, genome $\mathrm{n}$ was compared to any combinations of $\mathrm{n}-1$ genomes, and the number of identical 'core genes' and 'strain-specific genes' (specific for strain $\mathrm{n}$ ) were counted for each $\mathrm{n}$. According to the approach suggested, when all genomes are compared to $\mathrm{n}$ other genomes $(n=1, . ., \mathrm{N})$, this would result in $32 ! /[(n-$ $1) ! \cdot(32-n) !]$ possible combinations (for each $\mathrm{n}$ ) of drawing $\mathrm{n}$ genomes out of the pool of 32 genomes. Consequently, for 16 or 17 genomes ( $n=17,18$ in above formula), a total of 9.62 billion possible combinations exists. To reduce computational time, we lowered the number of combinations by randomly selecting 3,200 different combinations (or the maximum number of combinations; 3,200 is dividable by 32 , which ensures that all genomes are used an equal number of times 
as blast template for each $\mathrm{n}$ ) with an equal distribution among query genome. This was repeated ten times and an exponential decay function was fitted to each of these repeats. The decay function suggested by Tettelin et al [3] was first applied:

$$
F(n)=\kappa \cdot \mathrm{e}^{(-\mathrm{n} / \tau)}+g
$$

where $g$ equals the number of 'core genes' or 'specific genes', while $\kappa$ and $\tau$ are free parameters for amplitude of exponential decay. The speed at which $\mathrm{F}(\mathrm{n})$ converges was found to fit the data for 'strain-specific genes' satisfactorily, while a modified decay function with the double square root of $\mathrm{n}$ was found to fit the 'core genes' data better (lower sum of squared errors):

$$
F(n)=\kappa \cdot \mathrm{e}^{(-\mathrm{sqrt}(\operatorname{sqrt}(\mathrm{n})) / \tau)}+g
$$

\section{Strain selection, DNA preparation and hybridization} Control strain K-12 MG1655 was kindly provided by Flemming G Hansen (CBS, BioCentrum-DTU, The Technical University of Denmark) and genomic DNA from control strain O157:H7 EDL933 was kindly provided by Camilla Sekse (Norwegian Veterinary school, Oslo). As test strains, Kurt Zimmermann from Symbiopharm (Herborn, Germany) supplied four probiotic $E$. coli isolates, designated G1/2, G3/10, G4/9 and $\mathrm{G}_{5}$, from their commercially available Symbioflor2 product. G1/2 has previously been serotyped as O rough:K-:Hand $\mathrm{O}$ rough:H-, G3/10 as $\mathrm{O} 35,129: \mathrm{K}-\mathrm{H}-, \mathrm{G}_{4} / 9$ as $\mathrm{O}$ rough:K-:H-, and $\mathrm{G} 5$ as O rough:K-:H-.

All test strains were grown overnight in Luria-Bertani (LB) broth with continuous agitation [27], and DNA was isolated as described previously [28]. The genomic DNA was labeled with cy3 or cy5 and hybridized to NimbleGen custom arrays according to NimbleGen standard protocols for CGH (prepared and hybridized by NimbleGen (Madison, Wisconsin USA)). The raw data are available from the Gene Expression Omnibus (GEO) database [29] with series accession number GSE8595.

\section{Analysis methods}

The probes were mapped to each gene group including position according to the design and analyzed as described previously [2] with minor modifications. Briefly, a positiondependent segmentation algorithm was employed to partition data points into present and absent sequence segments constituting any given gene. For this, we used circular binary segmentation [11] with default settings as implemented in DNAcopy developmental version 1.2.1 written for the R statistical language [30]. As recommended by the authors, the data were first smoothed and subsequently segmented. Segmentation was followed by merging the output with MergeLevels [12] with a fixed threshold at the standard deviation between segmented $\log _{2}$ intensities and observed $\log _{2}$ intensities, or the standard deviation of segmented $\log _{2}$ ratios.
Consequently, following noise reduction by segmentation and merging, the cutoff for $\log _{2}$ intensities was found at the merged value between these two distribution maxima with the least segments assigned to it. All segments with merged values above this cutoff were predicted as present. Since ratios are calculated only for genes present in the control sample, and given the likely high similarity between a test sample and control sample of the same species, most genes are assumed present. Consequently, here the present level was estimated as the merged level to which most segments had been assigned. Moreover, for a gene to be called present, at least $90 \%$ of its probes should be found to be present. Accordingly, the samples were both analyzed individually as $\log _{2}$ intensities and combined with the appropriate control experiment, as $\log _{2}$ ratios.

Atlases were created using the GeneWiz software [31]. The blast atlases were constructed as described previously [32].

\section{Abbreviations}

aCGH, comparative genomic hybridization; FDR, false discovery rate.

\section{Authors' contributions}

$\mathrm{HW}$ and PFH designed the microarray. HW performed experimental work, analyzed the data and drafted the manuscript. DWU collected the genome sequences and supervised the project. TMW contributed with biological insight into $E$. coli pathogenicity. All authors edited and approved the final manuscript.

\section{Additional data files}

The following additional data are available with the online version of this paper. Additional data file 1 is a table providing a ranked list of each Symbioflor2 isolate's similarity to chip design strains. Additional data file 2 contains complete lists of annotated genes found in each of the four Symbioflor2 isolates but not in the MG1655 control strain. Additional data file 3 contains a detailed description of the microarray design.

\section{Acknowledgements}

The authors are grateful for support from the Danish Research Councils, as well as The Danish Center for Scientific Computing. We also wish to thank Flemming G Hansen, Kurt Zimmermann and Camilla Sekse for contributing E. coli strains. We would also like to acknowledge Carsten Friis, Aron C Eklund, Jon Bohlin and colleagues at CBS for many helpful contributions and discussions.

\section{References}

I. Dorrell N, Hinchliffe SJ, Wren BW: Comparative phylogenomics of pathogenic bacteria by microarray analysis. Curr Opin Microbiol 2005, 8:620-626.

2. Willenbrock H, Petersen A, Sekse C, Kiil K, Wasteson Y, Ussery DW: Design of a seven-genome Escherichia coli microarray for 
comparative genomic profiling. | Bacteriol 2006, I88:77| 3-772 I.

3. Tettelin H, Masignani V, Cieslewicz MJ, Donati C, Medini D, Ward NL, Angiuoli SV, Crabtree J, Jones AL, Durkin AS, et al.: Genome analysis of multiple pathogenic isolates of Streptococcus agalactiae : implications for the microbial "pan-genome". Proc Natl Acad Sci USA 2005, 102: I 3950-1 3955.

4. Medini D, Donati C, Tettelin H, Masignani V, Rappuoli R: The microbial pan-genome. Curr Opin Genet Dev 2005, I 5:589-594.

5. Yang J, Nie H, Chen L, Zhang X, Yang F, Xu X, Zhu Y, Yu J, Jin Q: Revisiting the molecular evolutionary history of Shigella spp. J Mol Evol 2007, 64:7I-79.

6. Lan R, Reeves PR: Escherichia coli in disguise: molecular origins of Shigella. Microbes Infect 2002, 4: I I25-I I 32.

7. Larsen TS, Krogh A: EasyGene - a prokaryotic gene finder that ranks ORFs by statistical significance. BMC Bioinformatics 2003 , 4:21

8. Nielsen $\mathrm{P}$, Krogh A: Large-scale prokaryotic gene prediction and comparison to genome annotation. Bioinformatics 2005, 21:4322-4329.

9. Tannock GW: Molecular assessment of intestinal microflora. Am J Clin Nutr 200I, 73:4I0S-414S.

10. Hartl DL, Dykhuizen DE: The population genetics of Escherichia coli. Annu Rev Genet 1984, 18:31-68.

II. Olshen $A B$, Venkatraman ES, Lucito R, Wigler M: Circular binary segmentation for the analysis of array-based DNA copy number data. Biostatistics 2004, 5:557-572.

12. Willenbrock $H$, Fridlyand J: A comparison study: applying segmentation to array CGH data for downstream analyses. Bioinformatics 2005, 21:4084-4091.

13. Perna NT, Plunkett G 3rd, Burland V, Mau B, Glasner JD, Rose DJ, Mayhew GF, Evans PS, Gregor J, Kirkpatrick HA, et al.: Genome sequence of enterohaemorrhagic Escherichia coli O I57:H7. Nature 200I, 409:529-533.

14. Roos V, Nielsen EM, Klemm P: Asymptomatic bacteriuria Escherichia coli strains: adhesins, growth and competition. FEMS Microbiol Lett 2006, 262:22-30.

15. Damian M, Usein CR, Tatu-Chitoiu D, Palade AM, Popovici N Ciontea S, Nica M, Grigore L: Incidence of virulence-encoding genes among enteric Escherichia coli strains isolated from healthy subjects. Roum Arch Microbiol Immunol 2005, 64:34-38.

16. Bettelheim KA, Kuzevski A, Gilbert RA, Krause DO, McSweeney CS: The diversity of Escherichia coli serotypes and biotypes in cattle faeces. J Appl Microbiol 2005, 98:699-709.

17. Schierack $P$, Steinruck $H$, Kleta S, Vahjen W: Virulence factor gene profiles of Escherichia coli isolates from clinically healthy pigs. Appl Environ Microbiol 2006, 72:6680-6686.

18. Chen Q, Savarino SJ, Venkatesan MM: Subtractive hybridization and optical mapping of the enterotoxigenic Escherichia coli HI0407 chromosome: isolation of unique sequences and demonstration of significant similarity to the chromosome of E. coli K-I 2. Microbiology 2006, I 52: I04I-I054.

19. Chen SL, Hung CS, Xu J, Reigstad CS, Magrini V, Sabo A, Blasiar D, Bieri T, Meyer RR, Ozersky $P$, et al: Identification of genes subject to positive selection in uropathogenic strains of Escherichia coli : a comparative genomics approach. Proc Natl Acad Sci USA 2006, 103:5977-5982.

20. Altschul SF, Gish W, Miller W, Myers EW, Lipman DJ: Basic local alignment search tool. I Mol Biol 1990, 2 I 5:403-410.

21. Thompson JD, Higgins DG, Gibson T]: CLUSTAL W: improving the sensitivity of progressive multiple sequence alignment through sequence weighting, position-specific gap penalties and weight matrix choice. Nucleic Acids Res 1994, 22:4673-4680.

22. Wernersson R, Nielsen HB: OligoWiz 2.0 - integrating sequence feature annotation into the design of microarray probes. Nucleic Acids Res 2005, 33:W6I I-6I5.

23. Schneider TD, Stephens RM: Sequence logos: a new way to display consensus sequences. Nucleic Acids Res 1990, 18:6097-6I00.

24. Hughes TR, Mao M, Jones AR, Burchard J, Marton MJ, Shannon KW, Lefkowitz SM, Ziman M, Schelter JM, Meyer MR, et al.: Expression profiling using microarrays fabricated by an ink-jet oligonucleotide synthesizer. Nat Biotechnol 200I, 19:342-347.

25. Altschul SF, Madden TL, Schaffer AA, Zhang J, Zhang Z, Miller W, Lipman DJ: Gapped BLAST and PSI-BLAST: a new generation of protein database search programs. Nucleic Acids Res 1997, 25:3389-3402.

26. O'Donovan C, Martin MJ, Gattiker A, Gasteiger E, Bairoch A, Apweiler R: High-quality protein knowledge resource: SWISS-PROT and TrEMBL. Brief Bioinform 2002, 3:275-284.
27. Sambrook J, Fritsch EF, Maniatis T: Molecular Cloning: a Laboratory Manual 2 nd edition. Cold Spring Harbor, NY: Cold Spring Harbor Laboratory Press; 1989.

28. Grimberg J, Maguire S, Belluscio L: A simple method for the preparation of plasmid and chromosomal E. coli DNA. Nucleic Acids Res 1989, 17:8893.

29. Barrett T, Edgar R: Gene expression omnibus: microarray data storage, submission, retrieval, and analysis. Methods Enzymol 2006, 4I I:352-369.

30. Bioconductor [http://www.bioconductor.org]

31. Pedersen AG, Jensen LJ, Brunak S, Staerfeldt HH, Ussery DW: A DNA structural atlas for Escherichia coli. I Mol Biol 2000, 299:907-930.

32. Hallin PF, Binnewies TT, Ussery DW: Genome update: chromosome atlases. Microbiology 2004, I 50:309|-3093.

33. Zoomable Hybridization and Blast Atlas for 'Characterization of Probiotic Escherichia coli Isolates Using a Novel Pangenome Microarray' [http://www.cbs.dtu.dk/services/ GenomeAtlas/suppl/zoomatlas/?zpid=ecoli_pangenome]

34. NCBI GenomeProjects [http://www.ncbi.nlm.nih.gov/genomes/ Iproks.cgi]

35. EasyGene I.2 [http://servers.binf.ku.dk/cgi-bin/easygene/search] 